\title{
Biological Control of Chestnut Blight in Portugal
}

\author{
L.M. Martins ${ }^{\mathrm{a}}$ and J.P. Castro \\ Univ. Tras-os-Montes Alto Douro \\ CITAB \\ Vila Real \\ Portugal
}

\author{
M.E. Gouveia \\ Instituto Politecnico de Qta Sta Apolónia \\ Bragança \\ Portugal
}

Keywords: Cryphonectria parasitica, vc type structure, biological control application

\begin{abstract}
Hypovirulence is a specific biological control method of chestnut blight, a lethal disease of American and European chestnut. The causal pathogen of chestnut blight is Cryphonectria parasitica, a fungus of Asian origin and an A2 quarantine organism in Europe. The disease has been reported since 1990 in Portugal, one of the last European countries where the pathogen was introduced. The chestnut blight fungus is now well established and widespread in Portugal with fast expansion in all chestnut regions. Biological control with hypovirulent strains of $C$. parasitica is considered an efficient means to control the disease and improve chestnut recovery. One of the goals of this study is to apply hypovirulence as a biological method for chestnut blight control and produce a solid scientific base to extend the treatment method over the country's chestnut areas. A successful biological control program will have a very high practical impact on crop productivity and on the social perception of applied research.

Field records and studies included parameters related to trees (dendrometric parameters and plant health status) and the physical characteristics of the plots (type of soil, exposure, geographic coordinates, age of trees, actual management of soil, etc.). Other scientific issues related to population structure of the pathogen include evolutionary forces that are present or dominate at population level (clonality, selfing, self-incompatibility, vc type structure, vc type segregation, CHV presence, $\mathrm{CHV}$ species). One of the goals of this study is to generate data that can answer questions dealing with the effect of the founder population and the driving forces on actual and future chestnut populations. The study also can produce an innovative and a realistic approach that has potential for large and extended field application with a positive impact on chestnut production.
\end{abstract}

\section{INTRODUCTION}

In spite of the decline of chestnut areas from 80,000 ha in the 1950 s to 45,000 ha in 2013, chestnut production remains crucial in Portugal. Chestnut production is most important on Denomination Protected Regions: Castanha da Padrela, Castanha da Terra Fria, and Soutos da Lapa. However, there are biotic constraints that impact chestnut production; these include two diseases of chestnut, ink disease (Phytophthora cinnamomi) and chestnut blight (Cryphonectria parasitica).

Chestnut blight was first detected in Europe in 1938 but it was not introduced to Portugal until the 1990s. The disease has expanded rapidly in all chestnut growing regions in Portugal. Recent surveys in areas where the disease was previously recognized revealed an increase in disease incidence ranging from 40-100\% (Azevedo et al., 2010; Bragança et al., 2009). A large number of newly infected sites, coupled with increased disease resistance has greatly increased the loss in chestnut fruit production has led to the decline and death of many chestnut trees.

Biological control utilizing hypovirulence, a virus-mediated attenuation of fungal pathogenesis, is an efficient mean to control the disease and improve chestnut recovery (Heiniger and Rigling, 1994; Robin and Heiniger, 2001; Cortesi et al., 1996).

almartins@utad.pt 
Hypovirulent strains of $C$. parasitica are associated with the presence of an uncapsidated double-stranded dsRNA virus in the cytoplasm of $C$. parasitica (Anagnostakis and Day, 1979; Dawe and Nuss, 2001).

In Portugal, two decades since the chestnut blight fungus was introduced, hypovirulence has been reported recently (Gouveia et al., 2010; Bragança et al., 2007). The Portuguese $C$. parasitica population is characterized by general low ve type diversity (Bragança et al., 2007) and in the northeast, many sub-populations have only one vc type. The dominant vc type is EU-11, which is somewhat rare outside of Portugal and is present only in some sites in Italy (Cortesi et al., 1996) and France (Robin et al., 2000).

The success of hypovirulence depends on population structure relative to the system of vegetative compatibility (vc types) (Heiniger and Rigling, 1994; Bissenger et al., 1997). The low diversity of vc types in Portugal infers potential success from hypovirulence release. Hypovirus has no extracellular phase; therefore, there are two means of dissemination: via conidia and anastomosis. To introduce the hypovirus by mass application of hypovirulent strains, several factors have to be considered. First, in order to have efficient biological control, the risk of increasing genetic diversity must be minimized. To accomplish this, only strains of the dominant ve type at the site or region should be used in the field. Second, strains of the dominant mating type should be used to reduce sexual recombination. Mating type proportion is a key factor for sexual reproduction and demographic structure of the population that may be altered by mass introduction of different mating type individuals.

In order to address the ecosystem-based approach, biological control is divided in three research lines:

1. Chestnut blight dispersal and Portuguese $C$. parasitica population characterization;

2. Field application of hypovirulent strains in selected sites;

3. MALTI-TOF MS identification of C. parasitica hypovirulent and virulent strains.

In research line 1, a Geographical Information System (GIS) was incorporated for accurate identification of geographical coordinates of chestnut groves and stands and affected trees. This information was critical for spatial reference of trees and for geostatistic studies.

The aim of this study was to evaluate the global evaluation of chestnut blight severity, its incidence and spatial spread. This information was fundamental to provide a solid base for future analysis of hypovirulence applications in the field.

\section{MATERIALS AND METHODS}

Based on initial foci and sites where chestnut blight was largely reported, a survey of chestnut trees based on one systematic spatial grid was performed. This study was limited to one site located in Parada, Bragança.

Field records included parameters related to trees (dendrometric parameters; plant health status; age of trees) and the physical characteristics of the plots (type of soil; exposure; geographic coordinates; actual management of soil, etc.). The affected trees were based on a $1-5$ scale of disease severity $(1=$ healthy tree; $5=$ dead tree). Cankers were classified and assessed for the presence of healing cankers (production of callus tissue and reduced sporulation), an indication of hypovirus infection. Parameters related to health status were also georeferenced (GPS) allowing for later analysis of the spread of the pathogen using geostatistical methods.

One geostatistical approach used a variography to analyse the spatial dependence of disease incidence. The semivariogram is a graphical representation of similarity (semivariance) of data pairs, in this case the health condition of a pair of trees, as a function of the lag distance (h), i.e., the distance between each data pair. As paired data values become less similar, the semivariogram increases in value (Isaaks and Srivastava, 1989; Pannatier, 1996). In the semivariogram, it is possible to observe the following parameters: a) the sill, semivariance value at model plateau, equal to the sample variance; b) the nugget, semivariance value at the origin; and c) the range as the distance at which the sill is reached, and the pairs become uncorrelated (Jia et al., 2008; Mulla, 2008; Shukla et al., 
2004; Matheron, 1962). The spatial dependence can be classified assuming the nugget as a percentage of the sill: $<25 \%$ (strong), between 25 and $75 \%$ (moderate), and $>75 \%$ (weak spatial dependence) (Cambardella et al., 1994).

\section{RESULTS AND DISCUSSION}

Field observations showed a sharp decline in tree health (Fig. 1). In almost all sites, ink disease was observed, although it was observed with greater intensity in areas of lower latitude (Fig. 2). Physical characteristics of the chestnut plots, 1 (soil depth, slope, exposure and actual management) were not found to be important factors relative to the incidence of ink disease (Fig. 3). This is partially explained in that P. cinnamomi zoospores and clamidospores are mobilized in the soil and transported to chestnut roots via water channels in the soil Cultural practices coupled with the hot, dry summer of 2013 also may have factored into the chestnut mortality.

Using a geostatistical approach, it was found that trees were in decline due to the incidence of chestnut blight (Fig. 4). In some plots, as had been notice in other regions, the disease affected more than $40 \%$ of the trees.

Field-collected $C$. parasitica cankers are used to gain an understanding of the fungal population in Portuguese stands and groves of chestnut from these cankers, the causal fungus is isolated, purified and maintained in culture. Future characterization will include vc typing of $C$. parasitica, DNA fingerprinting (the microsatellite genetic markers) Eric-PCR and Rep-PCR. and the presence of $C$. parasitica $\mathrm{CHV}$ infected strains also will be assessed (Davis et al., 2005).

\section{ACKNOWLEDGEMENTS}

Thanks to PTDC/AGR-PRO/120063/2010 for financial support.

\section{Literature Cited}

Azevedo, J., Coelho, V., Castro, J.P., Spinola, D. and Gouveia, E. 2010. Spatial dynamics of chestnut blight disease at the plot level using the Ripley's K function. Proceedings of the IUFRO Landscape Ecology Working Group International Conference. Forest Landscapes and Global Change. September 21-27, 2010, Braganca, Portugal.

Bissegger, M., Rigling, D. and Heiniger, U. 1997. Population structure and disease development of Cryphonectria parasitica in European chestnut forests in the presence of natural hypovirulence. Phytopathology 87:50-59.

Bragança, H., Simões, S., Onofre, N., Tenreiro, R. and Rigling, D. 2007. Cryphonectria parasitica in Portugal - Diversity of vegetative compatibility types mating types and occurrence of hypovirulence. Forest Pathology 37:391-402.

Cambardella, C.A., Moorman, T.B., Novak, J.M., Parkin, T.B., Karlen, D.L., Turco, R.F., and Konopka, A.E. 1994. Field-scale variability of soil properties in Central Iowa Soil. J. Soil Sci. Soc. of America. Madison, WI. 58:1501-1511.

Cortesi, P., Milgroom, M.G. and Bisiach, M. 1996. Distribution and diversity of vegetative compatibility types in subpopulations of Cryphonectria parasitica in Italy. Micol. Res. 100:1087-93.

Gouveia, E., Coelho, V. and Monteiro, L. 2010. Potential of local hypovirulent strains of Cryphonectria parasitica for biological control of chestnut blight. Acta Hort. 866:443448.

Heiniger, U. and Rigling, D. 1994. Biological control of chestnut blight in Europe. Ann. Rev. Phytopathol. 32:581-99.

Isaaks, E.H. and Srivastava, R.M. 1989. An Introduction to Applied Geostatistics. Oxford University Press, New York. 572p.

Jia, X.H., Li, X.R., Zhang, J.G. and Zhang, Z.S. 2008. Analysis of spatial variability of the fractal dimension of soil particle size in Ammopiptanthus mongolicus' desert habitat. Environmental Geology. Springer Verlag, Berlin /Heidelberg.

Matheron, G. 1962. Traité de Géostatistique Appliquée. Editions Technip.

Mulla, D.J. 2008. Spatial Variability. Dept. Soil, Water and Climate. University of 
Minnesota, St. Paul, MN.

Pannatier, Y. 1996. Variowin: Software for Spatial Data Analysis in 2D. Springer-Verlag, New York.

Robin, C., Anziani, C. and Cortesi, P. 2000. Relationship between biological control, incidence of hypovirulence, and diversity of vegetative compatibility types of Cryphonectria parasitica in France. Phytopathology 90:730-737.

Robin, C., Lanz, S., Soutrenon, A. and Rigling, D. 2010: Dominance of natural over released biological control agents of the chestnut blight fungus Cryphonectria parasitica in southeastern France is associated with fitness-related traits. Biol. Control 53:55-61.

Shukla, M.K., Slater, B.K., Lal, R. and Cepuder, P. 2004. Spatial variability of soil properties and potential management classification of a Chernozemic field in lower Austria. Soil Science 169:852-860.

\section{Figures}

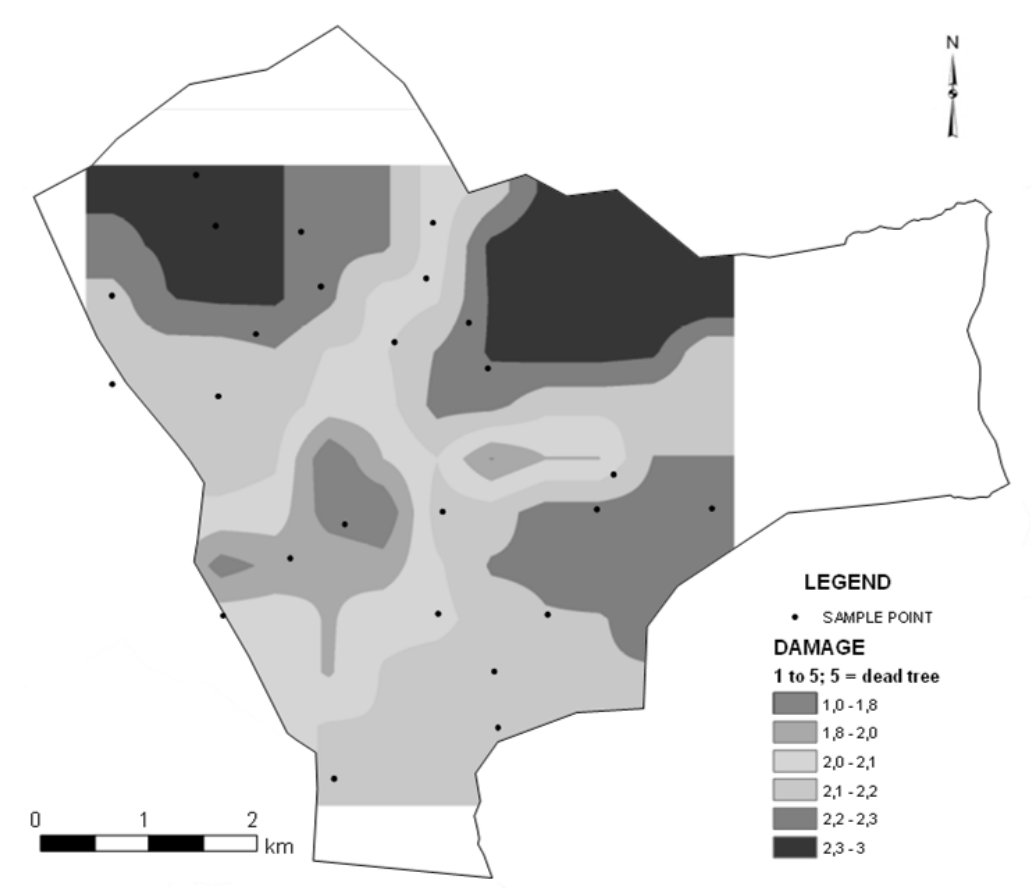

Fig. 1. Chestnut tree damage in study area in 2013. 


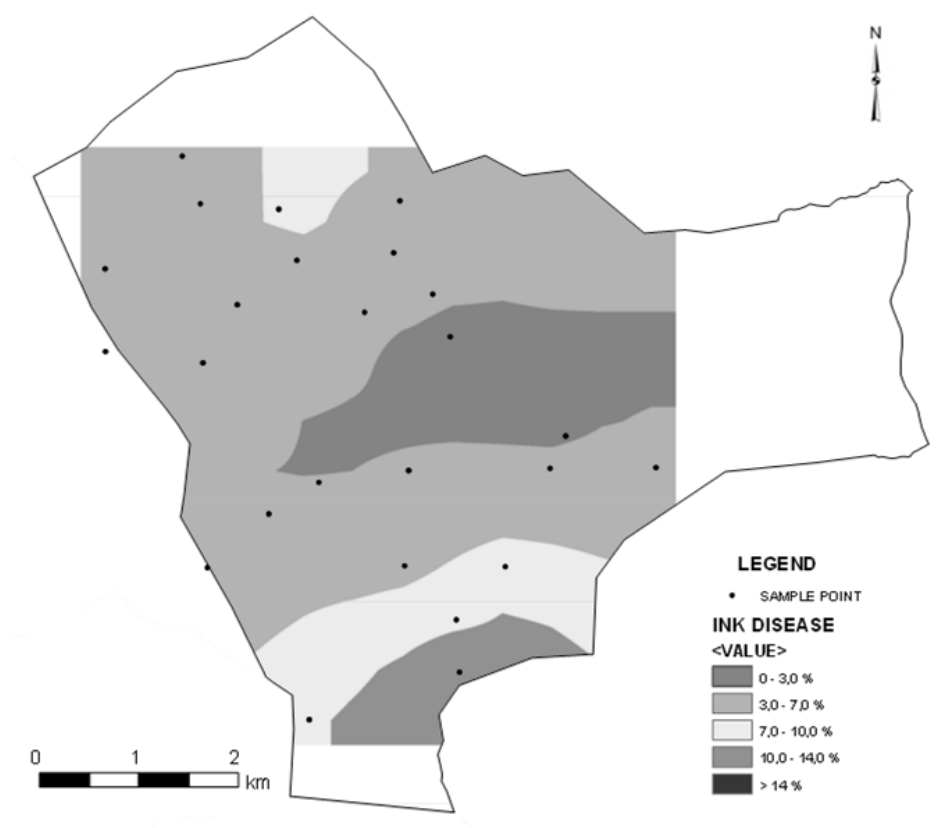

Fig. 2. Ink disease incidence in study area.

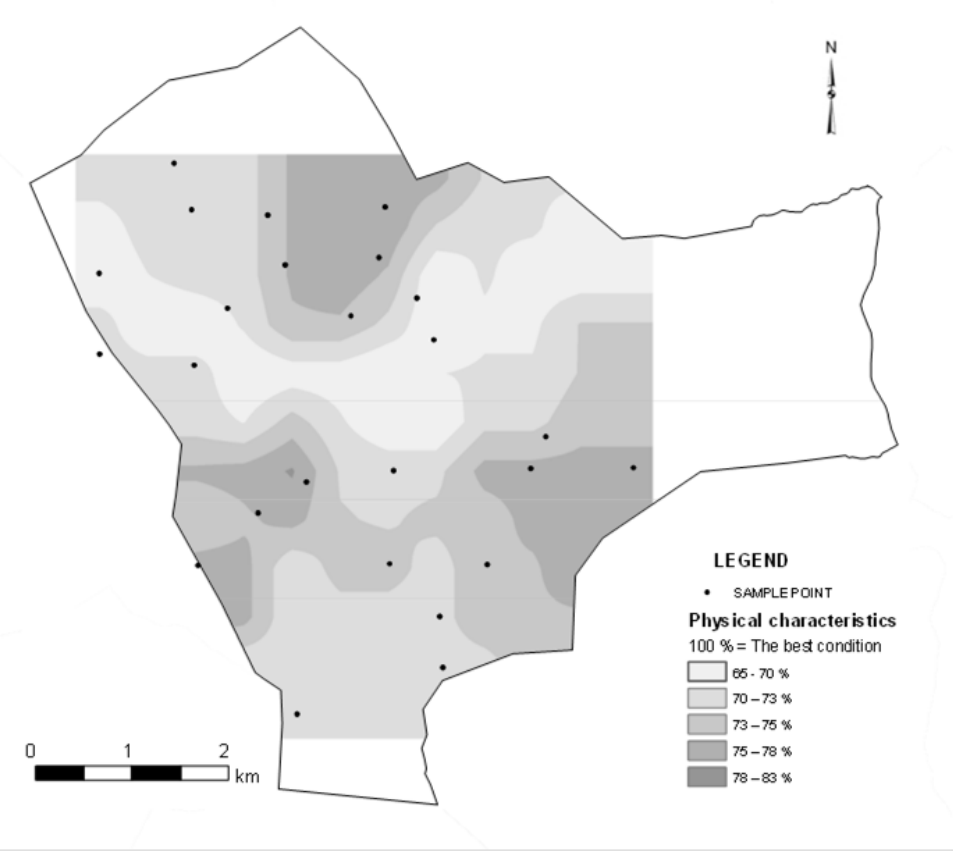

Fig. 3. Physical characteristics of the chestnut plots. 


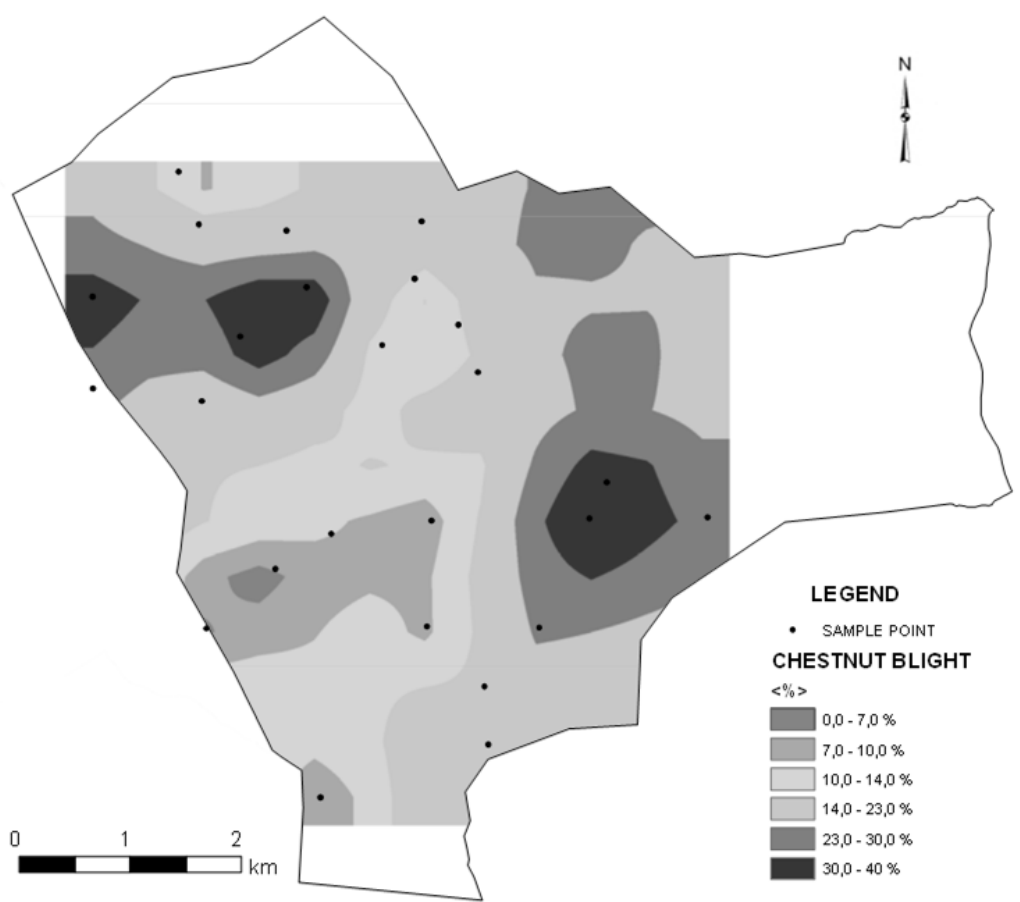

Fig. 4. Chestnut blight incidence in 2013. 